\title{
Nanostructure-initiator mass spectrometry: a protocol for preparing and applying NIMS surfaces for high-sensitivity mass analysis
}

\author{
Hin-Koon Woo ${ }^{1}$, Trent R Northen ${ }^{1,2}$, Oscar Yanes ${ }^{1}$ \& Gary Siuzdak ${ }^{1}$

\begin{abstract}
${ }^{1}$ Department of Molecular Biology, Scripps Center for Mass Spectrometry, The Scripps Research Institute, 10550 North Torrey Pines Road, La Jolla, California 92037, USA. ${ }^{2}$ Department of Genome Stability, Life Sciences Division, Lawrence Berkeley National Laboratory, 1 Cyclotron Road, Berkeley, California 94720 , USA. Correspondence should be addressed to G.S. (siuzdak@scripps.edu).
\end{abstract}

Published online 31 July 2008; doi:10.1038/nprot.2008.110

Nanostructure-initiator mass spectrometry (NIMS) is a new surface-based MS technique that uses a nanostructured surface to trap liquid ('initiator') compounds. Analyte materials adsorbed onto this 'clathrate' surface are subsequently released by laser irradiation for mass analysis. In this protocol, we describe the preparation of NIMS surfaces capable of producing low background and highsensitivity mass spectrometric measurement using the initiator compound BisF17. Examples of analytes that adsorb to this surface are small molecules, drugs, lipids, carbohydrates and peptides. Typically, NIMS is used to analyze samples ranging from simple analytical standards and proteolytic digests to more complex samples such as tissues, cells and biofluids. Critical experimental considerations of NIMS are described. Specifically, NIMS sensitivity is examined as a function of pre-etch cleaning treatment, etching current density, etching time, initiator composition, sample concentration, sample deposition method and laser fluence. Typically, NIMS surface preparation can be completed in less than $2 \mathrm{~h}$. Subsequent sample preparation requires 1-5 min, depending on sample deposition method. Mass spectrometric data acquisition typically takes 1-30 s per sample.

\section{INTRODUCTION}

The limitations of matrix-assisted laser desorption/ionization (MALDI) in small molecule analysis has prompted the investigations of new surface-based mass spectrometry techniques. Direct desorption/ionization (matrix-free) approaches have been developed to address the limitations of MALDI, while maintaining high throughput ${ }^{1}$. In particular, the uses of nanoscale materials such as nanostructured surface $e^{2-4}$, nanoparticles ${ }^{5-7}$, nanowires ${ }^{8}$ and nanotubes ${ }^{9,10}$ have found widespread applications to study a wide range of small molecules. Recently, we reported the versatile and high-sensitivity approach NIMS ${ }^{11}$. NIMS is a newly developed MS technique that uses nanostructured surfaces (e.g., porous silicon surface) to trap liquid 'initiator' materials (e.g., bis(heptadecafluoro-1,1,2,2-tetrahydrodecyl)tetramethyldisiloxane and compounds listed in Table 1), which can subsequently be released upon laser-induced surface heating. Analyte is absorbed on the initiator surface and desorbed upon initiator vaporization induced by laser irradiation. The initiator materials are transparent to UV laser and do not ionize, and analytes are not incorporated or cocrystallized with the initiator. These attributes represent fundamental differences from the MALDI approach. NIMS makes use of the nanostructured silicon surface, which is an efficient UVabsorbing semiconductor with a large surface area. Nanostructured silicon surface composed of $10-20 \mathrm{~nm}$ pores are produced by electrochemical etching of crystalline silicon using hydrofluoric acid (HF). As NIMS surfaces are not commercially available, these surfaces must be prepared by the user (following the protocol presented here), which involves chemicals including hazardous materials (acids and oxidizers) not required for commercial

TABLE 1 | Different compounds used as initiators for NIMS.

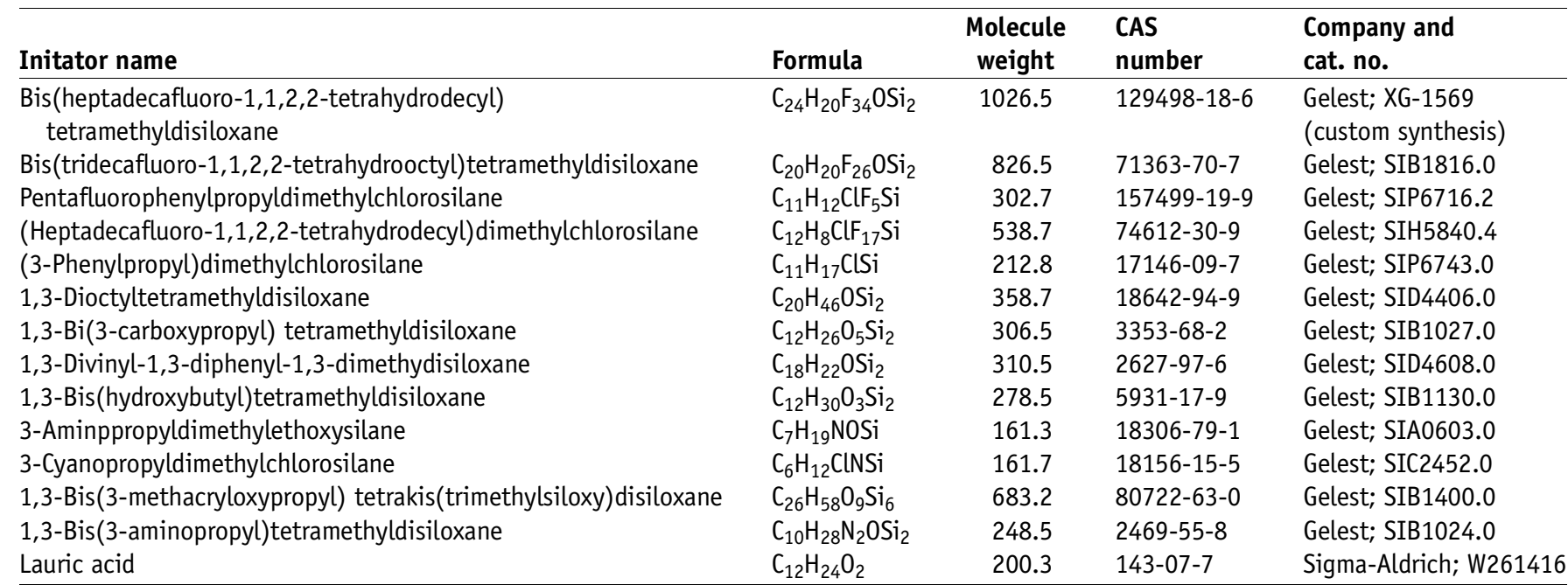


approaches such as MALDI. Because initiator does not ionize, it allows low-mass analytes to be examined with improved signal-tonoise $(\mathrm{S} / \mathrm{N})$ ratio and minimal background ion interference, extending the observable mass range to metabolites and drugs. In contrast to other matrix-free techniques, NIMS is a 'soft' desorption/ionization method with little or no analyte fragmentation.

A number of applications of NIMS have been presented, including direct detection of a wide range of biomolecules (e.g., small molecules, drugs, lipids and peptides) and a number of sample types: tissue imaging (e.g., mouse embryo tissue) and single cell analysis (e.g., cancer cell), as well as clinical characterization of biofluids with minimal sample preparation ${ }^{11}$. However, the performance of any NIMS application relies on its surface and sample preparation. Although it is not possible to cover the many applications of NIMS in one protocol, here we present a detailed procedure for the preparation of a low-background and high-sensitivity NIMS surface and its application for analyzing various types of analytes, which can be ionized through protonation or cationization from soluble samples such as biofluids. Examples are included to illustrate the performance of NIMS with appropriate preparation procedures and are easily extended to other sample types. There are a number of conditions which must be considered to achieve high sensitivity, including pre-etch surface cleaning treatment, etching time and current, sample application method, sample concentration and laser fluence. In particular, surface cleanliness is essential to provide the high sensitivity of NIMS; silicon surfaces used without proper cleaning procedures before electrochemical etching can result in low-mass background ions. NIMS activity is also significantly reduced when high laser fluences are used or if the analyte is spotted in high salt concentration or using an inappropriate sample application method. In the latter case, it is beneficial to spot the sample onto the surface a few times (i.e.,
Z-touch method) and then draw off the excess to form a thin layer of analyte on top of the initiator. This is in contrast to MALDI, which typically uses much higher laser fluence for desorption/ ionization, and its signal response depends on matrix concentration and cocrystallization with the analyte. Other considerations include the use of extensive etching time and current, which reduces surface sensitivity. Finally, it is shown that initiator selection can be used to improve NIMS analysis on carbohydrates.

\section{Designing a sample-analysis experiment}

Before spotting the analyte samples, it is important to consider the goals of the experiment (detection only or detection and quantification). It is often desirable to first confirm NIMS detection by making a dilution series of the molecule(s) into the representative background. For example, if it is desired to detect a drug in urine, it is best to first test the sensitivity of NIMS by 'spiking' the drug into the urine over a range of concentrations, as well as to determine if there are endogenous metabolites with similar mass. For quantification, although it is possible to make a standard curve from the dilution series (e.g., linear fit of the concentration versus peak intensity data $)^{11}$, it is typically more desirable to incorporate internal standards (surrogate molecules and/or stable isotopomers of the molecule of interest). In general, it is recommended to collect spectra under the same experimental conditions (laser intensity, number of laser shots, delayed extraction period and acceleration voltage, etc.) from at least three different positions $(5-10$ is preferred) on the chip. About 100 samples can be spotted (spotting volume $\sim 1.0 \mu \mathrm{l}$ each) on each NIMS chip. A NIMS chip can be reused; however, for the highest sensitivity measurements (presented here), new chips are preferred. Although new samples can be spotted and detected on used spots, there might be signal suppression and interference from the previously spotted samples.

\section{MATERIALS}

\section{REAGENTS}

•HF, 47-51\% assay (Fisher Scientific, cat. no. A513-500) ! CAUTION

Extremely toxic and highly corrosive.

-Sulfuric acid (Fisher Scientific, cat. no. A300-212) ! CAUTION Highly corrosive.

- Acetic acid (Sigma-Aldrich, cat. no. 320099) ! CAUTION Highly corrosive.

- Formic acid (Sigma-Aldrich, cat. no. 27001) ! CAUTION Highly corrosive.

- Hydrogen peroxide solution 30\% (wt/wt) in $\mathrm{H}_{2} \mathrm{O}\left(\mathrm{H}_{2} \mathrm{O}_{2}\right.$; Sigma-Aldrich, cat. no. 216763)! CAUTION Irritating to eyes and skin.

-Ethanol (Sigma-Aldrich, cat. no. 459828) ! CAUTION Highly flammable.

- Acetone (Fisher-Scientific, cat. no. A949) ! CAUTION Highly flammable.

- Acetonitrile (Fisher-Scientific, cat. no. A998) ! CAUTION Highly flammable and toxic.

- Methanol (Fisher Scientific, cat. no. A452)! CAUTION Highly flammable.

-Water $(>18 \mathrm{M} \Omega \mathrm{cm}$ ) from a nanopure water purification system (Barnstead NANOpure Infinity, Thermo Scientific)

- Bis(heptadecafluoro-1,1,2,2-tetrahydrodecyl)tetramethyldisiloxane

(BisF17; Gelest, cat. no. XG-1569 Custom synthesis)

- Pentafluorophenylpropyldimethylchlorosilane (PFP; Gelest, cat. no.

SIP6716.2)

- $\alpha$-Cyano-4-hydroxycinnamic acid ( $\alpha$-CHCA; Sigma-Aldrich,

cat. no. 476870)

- Phenylalanine (Sigma-Aldrich, cat. no. P-2126)

- Propafenone hydrochloride (MP Biomedicals, cat. no. 156383)

-1-Palmitoyl-sn-glycero-3-phosphocholine (Sigma-Aldrich, cat. no. L5254)

- Met-Arg-Phe-Ala acetate salt (MRFA; Sigma-Aldrich, cat. no. M1170)

- Bradykinin fragment 1-8 acetate hydrate (des-Arg'-Bradykinin;

Sigma-Aldrich, cat. no. B4397)

- Morphine was a gift from R.L. Fitzgerald and D. Herold
- Maltohexaose (Sigma-Aldrich, cat. no. 47873)

- Maltoheptaose (Sigma-Aldrich, cat. no. 47872)

- Bovine serum albumin (Sigma-Aldrich, cat. no. A7511)

- Dithiothreitol (DTT; Sigma-Aldrich, cat. no. D5545)

- Ammonium bicarbonate $\left(\mathrm{NH}_{4} \mathrm{HCO}_{3}\right.$; Sigma-Aldrich, cat. no. A6141)

- Iodoacetamide (IAA; Sigma-Aldrich, cat. no. I1149)

- Trypsin (Promega, cat. no. V5111)

- Ultra high purity (UHP) nitrogen (WestAir, cat. no. NIT300UHP)

- UHP oxygen (WestAir, cat. no. OX300UHP)

- Silicon wafer, p-type/Boron, $100 \mathrm{~mm}$ diameter, (100) orientation,

$525 \mu \mathrm{m} \pm 25 \mu \mathrm{m}$ thickness, single-side polished, resistivity $0.01-0.02 \Omega-\mathrm{cm}$ (Silicon Quest International and Silicon Materials) $\Delta$ CRITICAL All solvents should be obtained with the highest purity available. Slight contamination of any solvent will affect the sensitivity of NIMS.

EQUIPMENT

- A custom-made Teflon etching cell

- Gold foil (ESPI, 0.004" thick, 3N5 purity)

- Platinum wire (ESPI, 0.02" diameter, 3N5 purity)

- Ozone generator (Expotech USA Inc.)

- Diamond-tip scribe (Techni-Tool, cat. no. 758IE222)

- Stainless steel tweezer (Techni-Tool, cat. no. 758TW106)

- Stainless steel ruler (Techni-Tool, cat. no. 400PR124)

- DC Power supply, 0-30 V, 1.5 A (Protek Model No. 3033)

- Fiber-optic light source with a 250-W quartz halogen lamp (CUDA

Fiberoptics Model No. I-250)

- MALDI-TOF mass spectrometer (Voyager DE STR from Applied

Biosystems)

- Modified MALDI plate (Voyager MALDI sample plate from Applied

Biosystems, cat. no. V700401) 


\section{REAGENT SETUP}

Piranha solutions Mixture of sulfuric acid and hydrogen peroxide $\left(\mathrm{H}_{2} \mathrm{SO}_{4} / \mathrm{H}_{2} \mathrm{O}=2: 1\right)$ ! CAUTION Piranha is highly reactive and can become extremely hot when prepared; it is corrosive and irritating to the eyes, skin and respiratory tract. Always use glass containers as Piranha can melt plastics. We prepare this solution by adding the peroxide to the acid (violating the 'add acid to water' rule) by adding the peroxide solution very slowly while mixing the solution with a clean pasture pipette or glass rod. Mix the solution in a ventilated fume hood using proper gloves, lab coat and goggles. Safety guideline for handling Piranha solution can be found on several websites: http://web.princeton.edu/sites/ehs/labsafetymanual/cheminfo/piranha.htm; http://www.enma.umd.edu/LAMP/Sop/Piranha_SOP.htm; http://web.mit.edu/cortiz/www/ PiranhaSafety.doc; http://www.cise.columbia.edu/clean/process/Piranha_ Processing_Instruct.pdf; http://engineering.tufts.edu/microfab/index_files/ SOP/PiranhaClean_SOP.pdf.

Stock HF etching solution Prepare $25 \%$ HF etching solution in ethanol. ! CAUTION Extreme care should be taken in preparing the HF etching solution because of its toxicity and corrosiveness. All inhalation, ingestion or skin or eye contact should be strictly avoided. Preparation of HF etching solution should be conducted in a ventilated fume hood using proper nitrile gloves, lab coat and goggles. HF acid solution spills and burns can be neutralized and treated with $2.5 \%$ calcium gluconate gel. However, any direct contact with HF is potentially fatal and should be immediately treated by medical professionals.

Small molecules, drugs, lipids, peptides, carbohydrates and human urine Prepare phenylalanine, propafenone chloride, 1-palmitoyl-sn-glycero-3phosphocholine, MRFA and des-Arg ${ }^{9}$-bradykinin $\left(1 \mathrm{mg} \mathrm{ml}^{-1}\right)$ in $20 \%$ methanol in nanopure water. Prepare maltohexaose and maltoheptaose $\left(1 \mathrm{mg} \mathrm{ml}^{-1}\right)$ in nanopure water. These stock solutions are subsequently diluted using the corresponding solvents to the concentration needed. Urine samples are used promptly after diluting the urine 1:100 using nanopure water.

Matrix solution Prepare $\alpha$-CHCA $\left(10 \mathrm{mg} \mathrm{ml}^{-1}\right)$ in $50 \%$ acetonitrile in nanopure water, and add $0.1 \%$ formic acid.

Reagents for protein digest (i) $25 \mathrm{mM} \mathrm{NH}_{4} \mathrm{HCO}_{3}$ buffer; (ii) $10 \mathrm{mM}$ DTT in $25 \mathrm{mM} \mathrm{NH}_{4} \mathrm{HCO}_{3}$ buffer; (iii) $55 \mathrm{mM}$ IAA in $25 \mathrm{mM} \mathrm{NH}_{4} \mathrm{HCO}_{3}$ buffer; (iv) tryspin, $0.1 \mu \mathrm{g} \mu \mathrm{l}^{-1}$ in $\mathrm{H}_{2} \mathrm{O}$.

BSA digest preparation Prepare BSA $1 \mathrm{mg} \mathrm{m}^{-1}$ in $\mathrm{H}_{2} \mathrm{O}$. Boil in water for $1 \mathrm{~h}$ and take out $100 \mu \mathrm{l}$. Add $37.5 \mu \mathrm{l}$ of DTT and incubate the mixture at $60^{\circ} \mathrm{C}$ for 30 min. After cooling, add $17 \mu$ of IAA and incubate the mixture at room temperature $\left(20-25^{\circ} \mathrm{C}\right)$ in the dark for $30 \mathrm{~min}$. Afterward, add $75 \mu \mathrm{l}$ of DTT solution and allow it to remain at room temperature for $30 \mathrm{~min}$. Add $33 \mu \mathrm{l}$ of trypsin (with 1:30 enzyme-to-protein ratio), and incubate the digestion for $16 \mathrm{~h}$ at $37^{\circ} \mathrm{C}$. The reaction is stopped by adding $3 \mu \mathrm{l}$ of acetic acid.

\section{EQUIPMENT SETUP}

Setup of the etching cell A custom-made Teflon etching cell is composed of Teflon lid, Teflon base, gold foil electrodes, platinum wire, Kynar screws and caps (see Fig. 1). The etching cell should hold a chip with dimension about $3.3 \mathrm{~cm} \times 3.3 \mathrm{~cm}$, and the etched area is about $2.5 \mathrm{~cm} \times 2.5 \mathrm{~cm}=6.25 \mathrm{~cm}^{2}$. Etching cell and cutting tools cleaning Make sure the etching cell components and all other parts (tweezers, ruler and diamond-tip scribe) are clean. If parts are dirty, sonicate all parts in methanol for $30 \mathrm{~min}$.

Cutting the wafer Cut the wafer into appropriate size for etching chamber on a glass plate. Clean the glass plate using acetone and Kimwipes. Put the wafer on the cleaned glass plate with the polished side touching the glass surface. Draw lines on the back (unpolished side) of the wafer with the desired dimensions using a diamond-tip scribe and ruler. In the current etching cell setup, the dimension of the cut chip is about $33 \mathrm{~mm} \times 33 \mathrm{~mm}$. Typically, a $100-\mathrm{mm}$ diameter silicon wafer can be cut into four chips for etching. The drawn lines should be either parallel or perpendicular to the (110) plane if a (100) surface is used. The (110) plane is usually indicated as flats on the edge the wafer. Cleave the wafer according to the drawn lines (see Fig. 2).

$\triangle$ CRITICAL STEP When handling the silicon wafer, hold it from the corners or the edge of the chip. Avoid touching the surface of the chip.

Mass spectrometer MALDI-TOF mass spectrometer equipped with a $337 \mathrm{~nm}$ nitrogen laser (4 ns pulse duration). An acceleration voltage of $20 \mathrm{kV}$ is employed, and a delayed extraction period of 50-250 ns is used for optimal resolution. The laser intensity is attenuated by a neutral-density filter to optimize the $\mathrm{S} / \mathrm{N}$ ratio obtained for the mass spectral data. The outcome of this protocol should be the same with another commercial MALDI-TOF instrument (e.g., Bruker Daltonics, Thermo). The modified MALDI plates have a 1.5 inch $\times 1.5$ inch $\times 0.50 \mathrm{~mm}$ recess for the size and thickness of the silicon chips (see Fig. 2h).

Spectra analysis and MASCOT search Process spectra with baseline correction, noise filtering and monoisotopic peak list filtering using Data Explorer (Applied Biosystems). Calibrate the BSA digest spectra with internal peptide standard. Search the spectral mass peak lists using mascot peptide mass fingerprint with the following parameters: SwissProtein database, mammalian taxonomy, missed cleavage of 1 and \pm 100 p.p.m. peptide tolerance.
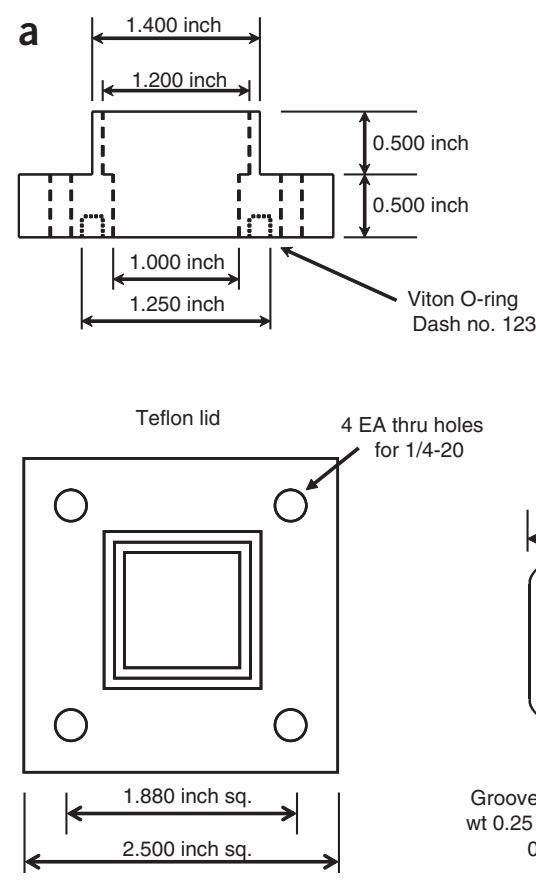
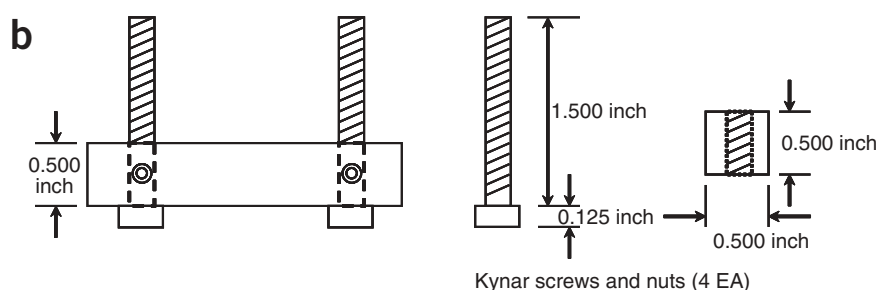

Kynar screws and nuts (4 EA)

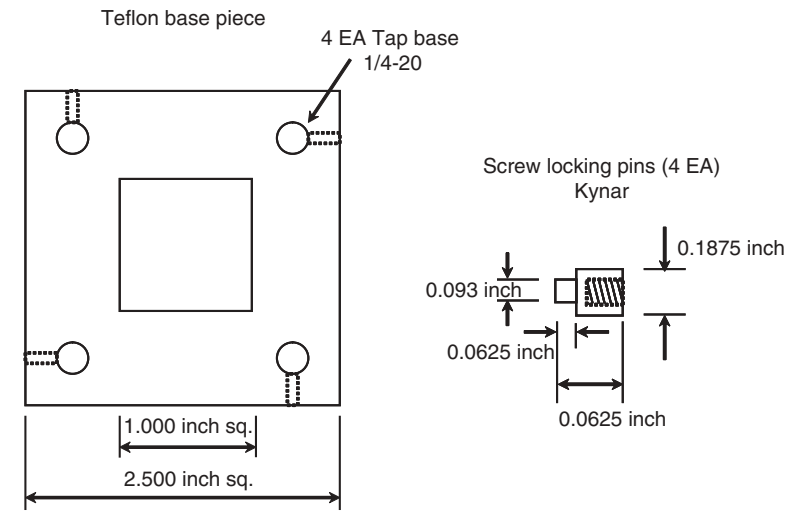

Figure 1 | Schematic diagram showing the dimensions of the etching chamber. (a) Teflon lid and Viton 0-ring; (b) Teflon base piece, Kynar screws and nuts. 
Figure 2 | Procedures for preparing an NIMS chip. (a) A glass plate for cutting the silicon wafer. A paper under the glass plate marked the diameter of the wafer $(100 \mathrm{~mm})$ and the dimension of the cut chip (about $33 \mathrm{~mm} \times$ $33 \mathrm{~mm}$ ). Place the wafer with polished side touching the glass plate and flats parallel to the grid line. (b) Draw lines on the back of the wafer according to the grid line using a diamond scribe and ruler. (c) Cleave the wafer according to the drawn lines. (d) A cut chip ready for Piranha solution cleaning. (e) A NIMS surface after the etching process (silicon wafer from Silicon Materials). (f) A NIMS surface with initiator BisF17 on it. (g) Blowing off the excess initiator. Note the change in color when initiator is blown off the nanostructure. (h) A NIMS chip mounted on a modified MALDI plate. Note that the modified MALDI plate is milled to offset the thickness of NIMS silicon wafer.

\section{PROCEDURE}

\section{Pre-etching treatment: silicon wafer cleaning using Piranha} solution

1) Soak the cut silicon wafer in the Piranha solution for 30 min. Use volume of Piranha solution just enough to submerge the wafer and always use glass containers.

I CAUTION Piranha solution is very energetic and potentially explosive. It will become very hot. Handle with great care and wear protective clothing.

$\triangle$ CRITICAL STEP It is recommended to prepare fresh Piranha solution for each use; the cleaning is more effective when the Piranha solution is fresh and hot.

2| Remove the wafer using a stainless steel tweezers.

3| Rinse the wafer chip with copious amount of nanopure water and blow dry with UHP nitrogen. Repeat this approximately 3-5 times.

$\triangle$ CRITICAL STEP Do not rinse the wafer using methanol or other organic solvents after Piranha solution cleaning, as mixing of Piranha solution with organic solvents may result in fire/explosion. Piranha solution cleaning removes the organics contaminants on the surface after soaking. Rinse surfaces with copious amounts of nanopure water and then rapidly blow-dry with a jet of UHP nitrogen.

$\triangle$ CRITICAL STEP Do not touch the cleaned inner portion of the chip (even with gloves on) as this may contaminate it.

$\triangle$ CRITICAL STEP A clean silicon surface before electrochemical HF etching is very important to make a low background noise and thus a high-sensitivity NIMS surface (see Fig. 3).

\section{Assembling the Teflon cell}

4| Assemble the chip holder by putting the 0-ring in the square groove of the Teflon lid (see Fig. 1a).

5| Place the silicon wafer with the polished side facing and touching the 0 -ring.

6| Place the gold foil on the unpolished side of the chip. A portion of the foil should stick out of the assembly.

7| Place the Teflon base (see Fig. 1b) of the assembly so that the chip and gold foil are sandwiched between the lid and the base.

8| Flip the whole assembly over so that the cell is faced with its right side up.

9| Finger-tighten and secure the assembly with screw caps, and tighten all of the screws gradually to prevent cracking the wafer.

$\triangle$ CRITICAL STEP Do not overtighten the screw caps. Overtightening the screw caps may crack the silicon wafer and cause HF leaking during the etching process.

\section{The etching process}

10| Attach positive $(+)$ or red electrode to the gold foil and then attach the negative $(-)$ or black electrode to the squareshaped platinum wire. 
11| Put the platinum wire on top of the chip inside the chamber.

12| Carefully add HF etching solution, just enough to submerge the platinum wire and the chip.

I CAUTION Extreme care should be taken in handling HF solution because of its toxicity and corrosiveness. All inhalation, ingestion or skin or eye contact should be strictly avoided.

13| Turn on the light source, which illuminates the unpolished side of the silicon wafer (optional).

$\triangle$ CRITICAL STEP Light illumination during etching is not critical for p-type silicon wafers. No effects on NIMS performance is observed when the light illumination is off during the etching process.

14 Turn on the power supply and apply a constant current of $300 \mathrm{~mA}$ (correspond to a current density of $48 \mathrm{~mA} \mathrm{~cm}^{-2}$ ) for 30 min onto the chip.

! CAUTION Etching of silicon wafer should be conducted in a ventilated fume hood using proper nitrile gloves, lab coat and goggles.

$\triangle$ CRITICAL STEP The performance of NIMS depends on the etching time and current density (see Fig. 4 and Table 2). Using a p-type silicon wafer with a resistivity of $0.01-0.02 \Omega-\mathrm{cm}$, it is recommended not to exceed an etching current of $48 \mathrm{~mA} \mathrm{~cm}^{-2}$ and an etching time of $40 \mathrm{~min}$.

\section{After etching}

15 Take out the platinum electrode and wash off any residual $\mathrm{HF}$ with methanol that may be on it. This is especially important for the negative $(-)$ or platinum electrode.

16 Carefully pour out the HF into a plastic beaker and wash the Teflon cell thoroughly with methanol and then properly dispose of the HF and wash solutions.

! CAUTION Dispose HF and wash solutions in a clearly labeled, chemically compatible container (e.g., polyethylene) with a

sealed lid. Glass, metal and ceramics are unsuitable containers.

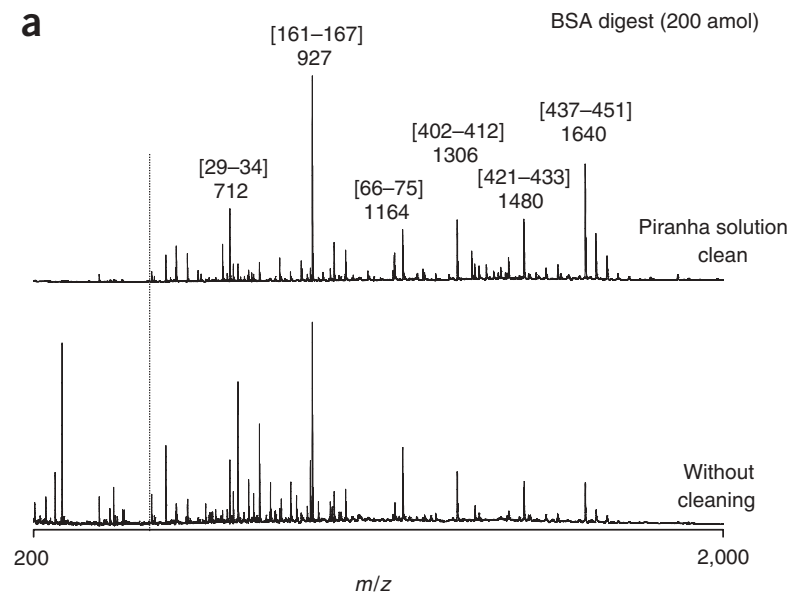

b
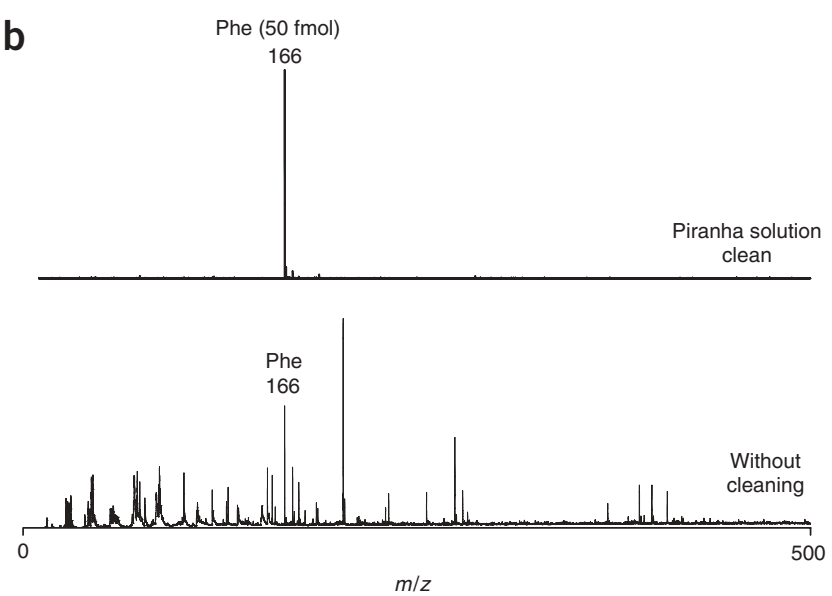

Figure 3 | Comparison of NIMS performance prepared with and without Piranha solution cleaning before electrochemical HF etching. (a) BSA digest $(200 \mathrm{amol})$ and $(\mathbf{b})$ phenylalanine $(50 \mathrm{fmol})$. The protein sequence coverage is $45 \%$ in a, upper and $43 \%$ in a, lower. The dashed line marked the $\mathrm{m} / \mathrm{z} 500$ position. Note the difference in background ions in the low mass region in a. NIMS surface prepared by Piranha solution cleaning give significantly lower background noise in the low mass region.

17| Dry the Teflon cell with UHP nitrogen.

18 Disassembly of the Teflon cell is the reverse of the assembly procedures stated in Step 4-9.

19| Wash the gold foil electrode thoroughly with methanol.

20| Carefully remove the etched chip with a pair of tweezers and wash off any HF residue with methanol. Dry the chip with UHP nitrogen. Repeat this approximately 3-5 times.

21| Dry the chip in a $100{ }^{\circ} \mathrm{C}$ oven for $\sim 5$ min.

22 0xidize the chip by directly blowing ozone on the chip for $5 \min$ (optional). Note that the ozone oxidization step is not critical. No significant effects on NIMS performance is observed if this step is skipped. The silicon surface is possibly air-oxidized quickly after the etching process.

- PAUSE POINT The etched chip can be stored in any clean and closed container under atmosphere pressure and room temperature for months without any significant change in performance. Initiator can be added about 60 min. before the mass analysis is performed. 


\section{Adding the initiator compound}

23| Add $\sim 100 \mu$ of initiator compound (BisF17) onto the whole etched surface of the chip (see Fig. 2e,f).

24| Let it soak in a glass Petri dish for approximately 30-60 min at room temperature.

$\triangle$ CRITICAL STEP Various initiator compounds can be used (see Table 1 for a list of initiator compounds). Initiator compounds can be specified to use for a particular type of analyte (see Fig. 5). In general, BisF17 is used because it is effectively trapped within the nanostructure and has the best performance for a wide range of molecules ${ }^{11}$. In addition, the relatively low vapor pressure of BisF17 makes it vacuum compatible with most MALDI instruments. In cases where more volatile initiators are used, cut the chip into smaller sections to prevent overwhelming the vacuum systems.

\section{Removing the excess of initiator compound}

25| Blow off excess initiator with a strong jet of UHP nitrogen pointed $\sim 30^{\circ}$ relative to the plane of the chip until only a thin film is left on the etched area of the chip (see Fig. $\mathbf{2 g}$ ).

26| Briefly place the chip in an $100{ }^{\circ} \mathrm{C}$ oven for about 3-5 s and blow off excess initiator with UHP nitrogen. Repeat these steps approximately 3-5 times until the chip appears completely dry. Particularly with BisF17, slight heating should cause the surface to change color as the initiator moves out of the nanostructures.

$\triangle$ CRITICAL STEP Do not completely dry the NIMS surface or leave too much initiator on the surface. The initiator trapped within the nanostructured silicon is required for the desorption/ionization process, yet too much excess may cause a vacuum (pumping) problem in the source chamber of the MALDI-TOF instrument.

- PAUSE POINT The freshly prepared NIMS surface can be stored in any clean and closed container under atmosphere pressure and room temperature for months without any significant change in performance. However, NIMS performance can decline after long periods under high vacuum, that is, several loading and unloading cycles of a NIMS chip in a mass spectrometer may result in the loss of initiator.

? TROUBLESHOOTING

\section{Mounting the chip}

27| Mount the chip onto a modified MALDI plate using clear adhesive tape (Scotch Transparent Tape 600) around the edges of the etched area of the chip (see Fig. $2 \mathbf{h}$ ).

$\triangle$ CRITICAL STEP The thickness recess of the modified MALDI plate is especially critical for NIMS performance. Adjust this thickness offset to be the same thickness as the NIMS wafer (typically $0.5 \mathrm{~mm}$ ). If the NIMS surface sticks out too much, it may damage the instrument; however, if it is sitting too deep inside the recess, the ion extraction efficiency may be reduced.

\section{Spotting samples}

28| Spot $0.5-1.0 \mu \mathrm{l}$ of samples with the desired concentration.

Note that about 100 sample spots (spotting volume $\sim 1.0 \mu \mathrm{l}$ each) can be deposited on each NIMS chip.

$\triangle$ CRITICAL STEP NIMS activity is sensitive to the sample concentration, although NIMS has high tolerances to salts and other contaminants. Surprisingly, NIMS performance is greatly reduced at high sample concentrations. There are generally two sample deposition methods that can minimize these effects. NIMS performance is best when a thin layer of analyte is applied.

TABLE 2 | Evaluation of NIMS.

\begin{tabular}{lcccc}
\hline & \multicolumn{4}{c}{ Etching current density $\left(\mathbf{m A ~} \mathbf{c m}^{-\mathbf{2}}\right)$} \\
\cline { 2 - 5 } & $\mathbf{8}$ & $\mathbf{1 6}$ & $\mathbf{3 2}$ & $\mathbf{4 8}$ \\
\hline Sequence coverage (\%) & $31 \pm 6$ & $35 \pm 7$ & $34 \pm 4$ & $42 \pm 4$ \\
No. of matched peptides & $23 \pm 4$ & $25 \pm 4$ & $25 \pm 3$ & $31 \pm 3$
\end{tabular}

BSA digest (200 amol) data for NIMS surfaces prepared using different etching current density (etching time was kept at $30 \mathrm{~min}$ ). BisF17 was used as the initiator compound. The mean value was calculated from the Mascot search results of ten individual spectra. Errors represent \pm 1 s.d. from the mean. Generally, thick samples such as those with high concentrations or salts content (i.e., biofluids) are best analyzed using the Z-touch method rather than the dried droplet method commonly employed in MALDI. Z-touch is performed by pipetting the sample droplets up and down 5-10 times before pipetting it off the surface. For samples with lower concentration or lesser salts content, directly depositing the sample on the NIMS surface and allowing it air-dried are recommended (see Fig. 6 ). In either case, a thin layer of analyte sample is deposited on the surface 
and the sample spot is usually not clearly shown on the monitor of the MALDI-TOF instrument screen, as opposed to the crystallized spot typically observed in MALDI-MS.

$\triangle$ CRITICAL STEP The concept of high and low concentration varies between samples (see Fig. $\mathbf{6 b}$ ). In general, if a thick sample layer/spot is seen on the NIMS surface or the MALDI-TOF instrument screen, its concentration is considered to be too high for NIMS analysis. Typically, NIMS gives the best performance if the sample layer/spot is barely or not visible at all on the surface.

\section{NIMS analysis}

29| Insert the modified MALDI target plate to be analyzed in the MALDI-TOF mass spectrometer.

30| Analyze the samples on a MALDI-TOF instrument operating in reflectron mode for mass range below 4,000 Da. Typically, 50-200 laser shots for each spot and a laser fluence of $10-40 \mathrm{~mJ} \mathrm{\textrm {cm } ^ { - 2 }}$ is recommended. The total number of laser shots is not critical and may be optimized for samples with different concentrations. For example, for an analyte with low concentration ( $<$ amol), too many laser shots will lead to noise accumulation after the analyte is depleted.

$\triangle$ CRITICAL STEP NIMS activity is very sensitive to the laser fluence used. In general, higher laser fluence will not give higher ion signals. The laser fluence typically employed in NIMS is significantly lower than MALDI (see Fig. 7). The uses of high or low laser fluence depends on the type of samples and may be optimized (see Fig. 8).

31 Randomly raster the target plate with the laser beam shooting around the analyte spots during the data acquisition process. When performing small molecule analysis $(\mathrm{m} / \mathrm{z}<500 \mathrm{Da})$, a shorter delayed extraction period ( $<150 \mathrm{~ns})$ and a higher acceleration voltage is recommended.

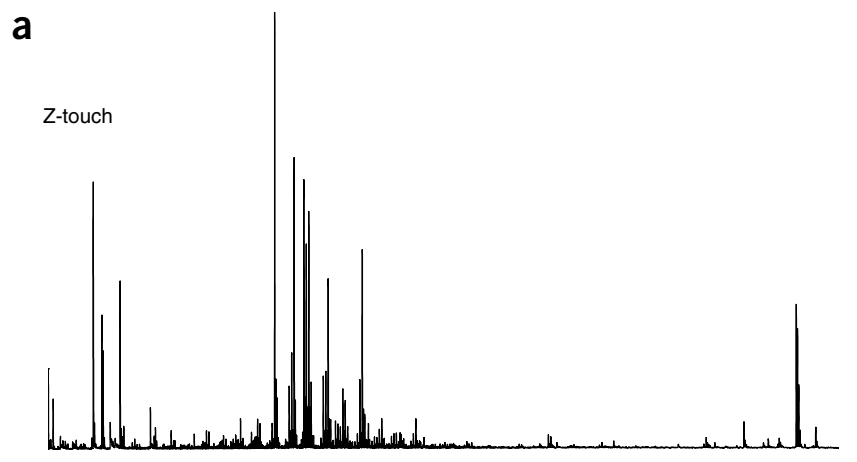

Dried droplet
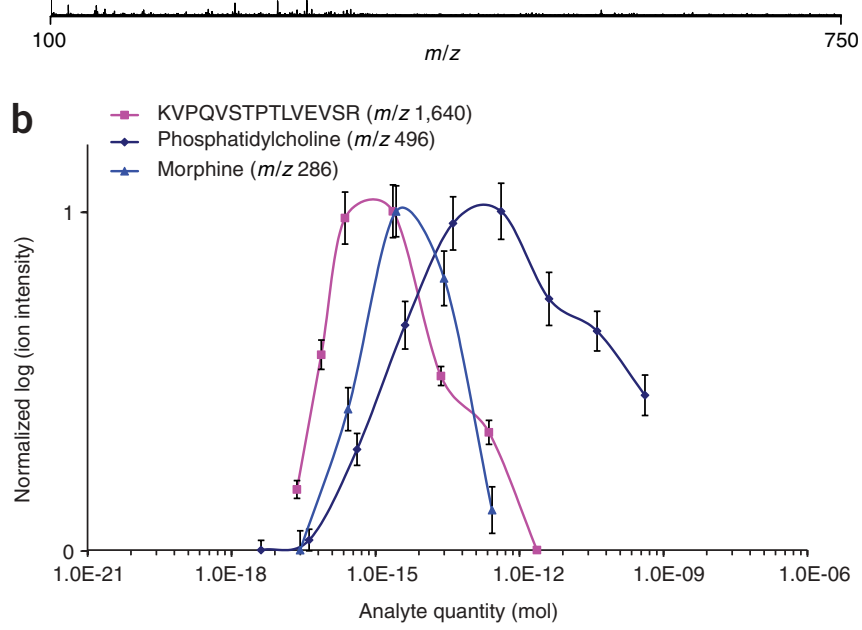

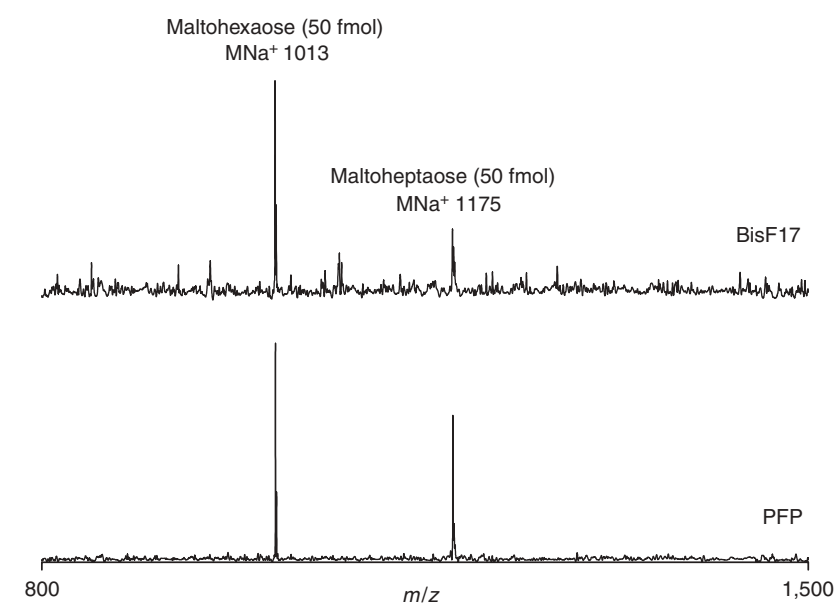

Figure 5 I Comparison of NIMS activity using different initiators for carbohydrates analysis. NIMS mass spectra obtained from a mixture of maltohexaose and maltoheptaose (50 fmol each) using BisF17 and PFP as initiators. The $\mathrm{S} / \mathrm{N}$ ratios measured are 34:1 (maltohexaose) and 10:1 (maltoheptaose) using BisF17. These $\mathrm{S} / \mathrm{N}$ ratios improve to $80: 1$ (maltohexaose) and 54:1 (maltoheptaose) when PFP is used. ? TROUBLESHOOTING

\section{TIMING}

Steps 1-3, silicon wafer pre-etch cleaning: 30-60 min Steps 10-14, the etching process: $30 \mathrm{~min}$ Steps 15-26, post-etching treatment: $50-80 \mathrm{~min}$ Step 28, sample deposition: $10 \mathrm{~s}$ to $5 \mathrm{~min}$, depending on whether Z-touch or dried droplet method is being used

\section{$?$ TROUBLESHOOTING}

Troubleshooting advice can be found in Table 3.

Figure 6 | Effect of sample application methods and sample concentrations on NIMS performance. (a) Comparison of mass spectra obtained from human urine using Z-touch and dried droplet deposition method. No signal was observed in the dried droplet deposition as urine forms a thick layer on top of the initiator. The Z-touch method is recommended when biofluids or samples with high concentration and salt contents are analyzed. (b) Sample quantity dependence of NIMS performance for different analytes: morphine, 1-palmitoyl-sn-glycero-3-phosphocholine and peptide KVPQVSTPTLVEVSR from BSA digest are spotted on the surface via dried droplet deposition method. NIMS activity reduces significantly when the sample concentration is high. The limits of detection are $500 \mathrm{amol}, 50 \mathrm{amol}$ and $100 \mathrm{amol}$ for morphine, 1-palmitoyl-sn-glycero-3-phosphocholine and KVPQVSTPTLVEVSR, respectively. Ion intensities from 10 individual spectra were used to calculate the mean. Ion intensity for each analyte is normalized to the corresponding maximum averaged intensity for comparison. Error bars represent \pm 1 s.d. from the mean. 
PROTOCOL

TABLE 3 | Troubleshooting table.

\begin{tabular}{llll}
\hline Step & Problem & Possible reason & Solution \\
\hline 26 & $\begin{array}{l}\text { Vacuum problem; source } \\
\text { chamber pressure is }\end{array}$ & Initiator is too volatile & Cut the chip into smaller pieces
\end{tabular}

Excessive initiator on the surface

$31 \quad$ Background ions signals are too high

\section{ANTICIPATED RESULTS}

This protocol for making NIMS chips results in surfaces capable of high sensitivity mass analysis of a wide range of molecules including small molecules, drugs, lipids, carbohydrates and protein digest. Reproducible results are obtained with silicon wafers purchased from two different companies (Silicon Quest International and Silicon Materials). In some cases, the silicon wafers have surface contaminants and need to be cleaned before etching using the sulfuric acid/hydrogen peroxide mixture 'Piranha'. We note that to obtain high-sensitivity measurement, it is important that the silicon surfaces, the necessary equipments/tools, containers and all the solvents used are extremely clean. Figure $3 a$ shows NIMS spectra of a tryptic digested BSA (200 amol) with different pre-etch cleaning procedures. The protein sequence coverage is $45 \%$ in Figure 3a (upper) with Piranha solution cleaning and $43 \%$ in Figure 3a (lower) with no cleaning. Although the percentage of protein sequence coverage is similar in both cases, there is a high level of noise in the low mass region $(<500 \mathrm{Da})$. The NIMS chip prepared with the pre-etch cleaning using the Piranha solution described in the protocol here gives
Remove excess initiator by heating up the surface briefly and blow off excess initiator. Repeat Steps 25 and 26

Prepare fresh Piranha solutions and repeat Step 1-3. Prolong the soaking in Piranha solutions up to $1 \mathrm{~h}$

Replace solvent with fresh, ultra-pure grade solvents and freshly cleaned containers

Reduce the laser fluence to appropriate energy level

Adjust the thickness offset of the MALDI plate with the same thickness as the NIMS wafer

Optimize the NIMS chip ion signal using some standard peptide before the analysis of the real samples

Use the Z-touch method for sample deposition instead of dried droplet

Use high laser fluence to remove excess material followed by analysis with lower laser fluence

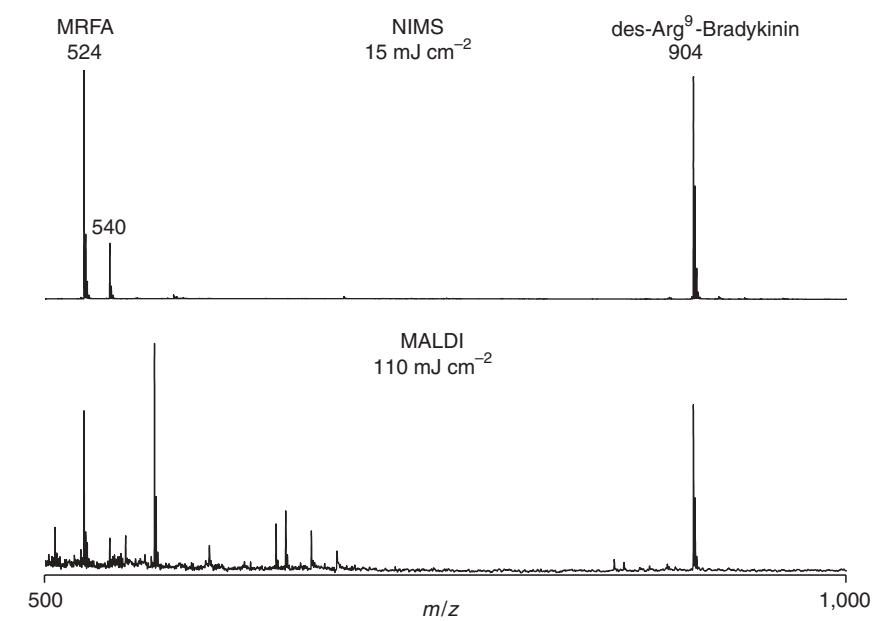

Figure 7 | A comparison of laser energy required to desorb/ionize tetrapeptide MRFA (50 fmol) and des-Arg ${ }^{9}$-bradykinin $(25 \mathrm{fmol})$ on NIMS and MALDI. The peak at $m / z 540$ is the oxidized form of MRFA. The typically laser fluence employed in NIMS is about $7 \times$ lower than MALDI. MALDI also gives more background peaks and lower $\mathrm{S} / \mathrm{N}$ ratio. $\alpha$-CHCA matrix was used in MALDI. 
significantly lower background ions in the low mass region. Figure $\mathbf{3} \mathbf{b}$ further illustrates the importance of pre-etch cleaning using phenylalanine $(50 \mathrm{fmol})$ as an example.

The effect of etching time and current density is shown in Figure $\mathbf{4}$ and Table 2. In general, the nanostructure depth is proportional to the etching time and current density. Using a current density of $48 \mathrm{~mA} \mathrm{~cm}^{-2}$, a minimum of 20-min etching time is required. Short etching time $(<20 \mathrm{~min})$ resulted in low $\mathrm{S} / \mathrm{N}$ ratio, whereas prolonged etching $(>40 \mathrm{~min}$ ) resulted in very fragile surface and the peeling of the nanostructured silicon surface and the whole chip collapsing if etching continues. Figure 4 shows the difference in performance for NIMS surface prepared at various etching times (etching current maintained at $48 \mathrm{~mA} \mathrm{~cm}^{-2}$ ) using propafenone ( $5 \mathrm{fmol}$ ). No significant effects on NIMS performance was observed when the etching time changed from 30 to $40 \mathrm{~min}$. With the etching time maintained at 30 min, changing the etching current density has similar effect as the etching time; low etching current density $\left(<32 \mathrm{~mA} \mathrm{~cm}^{-2}\right)$ resulted in low $\mathrm{S} / \mathrm{N}$ ratio. High etching current $\left(>48 \mathrm{~mA} \mathrm{~cm}^{-2}\right)$ also resulted in fragile silicon surface and the peeling of the surface after etching. The etching current density is typically kept at $48 \mathrm{~mA} \mathrm{~cm}{ }^{-2}$ because it gives higher peptide sequence coverage of protein digest (200 amol), as summarized in Table 2.

The use of specific initiators for certain types of analyte is demonstrated in Figure 5. Although BisF17 is a more universal initiator, the use of PFP provides better NIMS performance when analyzing carbohydrates. In Figure $\mathbf{5}$, higher $\mathrm{S} / \mathrm{N}$ is obtained for carbohydrate maltohexaose and maltoheptaose (50 fmol each) using PFP as an initiator. Figure 6 shows the relation of sample concentration, sample salt content and sample deposition method on NIMS analysis. Using human urine with high metabolite and salt content as an example (Fig. 6a), the Z-touch method demonstrates the utility and sensitivity of this sample deposition technique applied to biofluid analysis. No NIMS activity is observed using the conventional dried droplet method that forms a thick layer on the surface. The concentration dependence of NIMS activity with different types of analytes (drug, lipid and peptide) is shown in Figure $6 \mathbf{b}$. The dried droplet deposition method is used to demonstrate this effect. No ion signal is obtained when the sample concentration is too high. Figure $\mathbf{6 b}$ also shows that optimum NIMS activity varies with analyte type. It is worth mentioning that such signal reduction is not associated with the Z-touch method for any of the compounds tested so far.

The laser fluence used also affects NIMS performance and varies with sample type. A comparison of typical laser energy used in NIMS and MALDI is shown in Figure 7 using MRFA (50 fmol) and des- rrg $^{9}$-bradykinin $(25 \mathrm{fmol})$. NIMS requires significantly lower laser energy than MALDI. The laser fluence employed is critical and should be optimized for different types of analytes with specific concentration (Fig. 8). Similar to the concentration-dependent curve (Fig. 6b), Figure 8 shows that no NIMS activity is observed when the laser fluence used is too high.

ACKNOWLEDGMENTS This project was funded by DOE GTL Grants (MAGGIE and GeneMAP). This work was supported by the US Department of Energy Office of Science under contract no. DE-AC02-05CH11231 through the Genomics: GTL Research Program.

Published online at http://www.natureprotocols.com/ Reprints and permissions information is available online at http://npg.nature.com/ reprintsandpermissions/

1. Peterson, D.S. Matrix-free methods for laser desorption/ionization mass spectrometry. Mass Spectrom. Rev. 26, 19-34 (2007).

2. Wei, J., Buriak, J.M. \& Siuzdak, G. Desorption-ionization mass spectrometry on porous silicon. Nature 399, 243-246 (1999).

3. Nayak, R. \& Knapp, D.R. Effects of thin-film structural parameters on laser desorption/ionization from porous alumina. Anal. Chem. 79, 4950-4956 (2007).

4. Wada, Y., Yanagishita, T. \& Masuda, H. Ordered porous alumina geometries and surface metals for surface-assisted laser desorption/ionization of biomolecules: possible mechanistic implications of metal surface melting. Anal. Chem. 79, 9122-9127 (2007).
5. Wen, X.J., Dagan, S. \& Wysocki, V.H. Small-molecule analysis with siliconnanoparticle-assisted laser desorption/ionization mass spectrometry. Anal. Chem. 79, 434-444 (2007).

6. McLean, J.A., Stumpo, K.A. \& Russell, D.H. Size-selected (2-10 nm) gold nanoparticles for matrix assisted laser desorption ionization of peptides. J. Am. Chem. Soc. 127, 5304-5305 (2005).

7. Seino, T. et al. Matrix-free laser desorption/ionization-mass spectrometry using self-assembled germanium nanodots. Anal. Chem. 79, 4827-4832 (2007).

8. Go, E.P. et al. Desorption/ionization on silicon nanowires. Anal. Chem. 77, 1641-1646 (2005).

9. Xu, S.Y. et al. Carbon nanotubes as assisted matrix for laser desorption/ionization time-of-flight mass spectrometry. Anal. Chem. 75, 6191-6195 (2003).

10. Ren, S.F., Zhang, L., Cheng, Z.H. \& Guo, Y.L. Immobilized carbon nanotubes as matrix for MALDI-TOF-MS analysis: Applications to neutral small carbohydrates. J. Am. Soc. Mass Spectrom. 16, 333-339 (2005).

11. Northen, T.R. et al. Clathrate nanostructures for mass spectrometry. Nature 449, 1033 (2007). 